\title{
Bacterial Infection within the Root Canal: Lymphocytes, LPS, and the Inflammatory Response
}

\author{
Michel Goldberg* \\ Professor, Biomédicale des Saints Pères, Paris Descartes University, France \\ *Corresponding Author: Michel Goldberg, Professor, Biomédicale des Saints Pères, Paris Descartes University, France.
}

Received: June 18, 2019; Published: July 01, 2019

DOI: 10.31080/ASMI.2019.02.0288

The root canal flora of teeth with clinically intact crowns, but having necrotic pulps and diseased periapices, is dominated (> $90 \%$ ) by anaerobes usually belonging to the genera Fusobacterium, Porphyromonas (formerly Bacteroides), Prevotella (formerly Bacteroides), Eubacterium, and Peptostreptococcus. Spirochetes and fungi. They are also present in necrotic infected root canals. The intraradicular survival and pathogenic properties of the endodontic flora are influenced by a combination of factors, including:

1. Interactions with other micro-organisms in the root canal, to develop synergistically beneficial partners,

2. The ability to interfere with host defenses,

3. The release of lipopolysaccharides (LPS) and other bacterial modulins,

4. The synthesis of enzymes that damage host tissues.

Antibiotics are used in dental practice to prevent and cure regional or systemic infections. Microorganisms are the target of the antibiotic treatment.

LPS, also historically known as endotoxins form an integral part of Gram-negative cell walls. They are released during disintegration of bacteria after death and during multiplication and growth. The effects of LPS are due to their interaction with endothelial cells and macrophages. LPS not only signal the endothelial cells to express adhesion molecules but also activate macrophages to produce several molecular mediators such as the tumor necrosis factor- $\alpha($ TNF- $\alpha)$ and interleukins.

LPS are not the only bacterial degradation product that can induce mammalian cells to produce cytokines. Many proteins, certain carbohydrates, and lipids of bacterial origin are now considered as belonging to a novel class of 'modulins' that induce the formation of cytokine networks and host tissue pathology.

Microbial collagenase, hyaluronidase, fibrinolysins, and several proteases are examples of enzymes produced by endodontic mi- croorganisms. Microbes are also known to produce enzymes that degrade various plasma proteins involved in blood coagulation and other body defenses.

Polymorphonuclear leukocytess (PMN), lymphocytes, plasma cells, and monocytes/ macrophages play role in the defense systems. The three major classes of lymphocytes are the T-lymphocytes, B-lymphocytes, and the natural killer (NK) cells. The T- and B-lymphocytes are of importance in apical periodontitis.

The thymus-derived ( $\mathrm{T}$ ) cells have been designated after their effects or function. For instance, the T-cells working with B-cells have been known as T-helper/inducer (Th/i) cells, and those with direct toxic and suppressive effects on other cells have been named T-cytotoxic/suppressive (Tc/s) cells.

The B-lymphocytes directly responsible for antibody production are the bursa-equivalent (B) cells. Macrophages are phagocytic cells. Cytokines IL-1, TNF- $\alpha$ interferons (IFN), and growth factors contribute to the release of prostaglandins and leukotrienes.

Osteoclasts destroy bone and dental mineralized tissues. Epithelial cell rests are stimulated by cytokins and growth factors. They contribute to the formation of radicular cysts. Interleukins (IL-1, -6 , and -8 and tumor necrosis factor- $\alpha$ (TNF) $\alpha$, and IФN) are

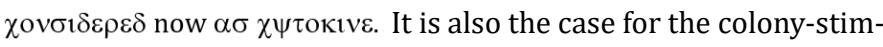
ulating factors (CSF).

Membrane lipids generate compounds that are intra- and inter cellular signals. Arachidonic acid, a 20-carbon polyunsaturated fatty acid, is released from membrane lipids and contribute to the formation of eicosanoids that mediate inflammatory response, Prostaglandins and leukotrienes are the two major groups of eicosanoids involved in inflammation. Enzymatic effector molecules are implicated in the destruction of the extracellular matrices. Topic application of a triple antibiotic paste consisting of metroni- 
dazole, ciprofloxacin, and minocycline has been shown to be very effective against the pathogens commonly found inside the root canal system.

Four major degradation pathways are recognized within and around teeth : 1) osteoclastic, 2) phagocytic, 3) plasminogen-dependent, and 4) metallo-enzyme regulated molecules. In addition, plasma cells produce antibodies [1].

The logical treatment of root canal has been to eliminate infection and exclude further infection of the canal $[2,3]$. Since the essential role of root canal microbes in both primary and post-treatment apical periodontitis has been well-recognized [4], the major treatment procedure should be the clinical management of problems associated with the control and elimination of infection [5].

\section{Bibliography}

1. Nair PNR. "Pathogenesis of apical periodontitis and the causes of endodontic failures". Critical Reviews in Oral Biology and Medicine 15.6 (2004): 348-381.

2. Vijayaraghavan R., et al. "Triple antibiotic paste in root canal therapy". Journal of Pharmacy and Bioallied Sciences 4 (2012): S230-S233.

3. Mohammadi Z and Abbott PV. "On the local application of antibiotics and antibiotic-based agents in endodontics and dental traumatology". International Endodontic Journal 42.7 (2009): 555-567.

4. Sato I., et al. "Sterilization of infected root-canal dentine by topical application of a mixture of ciprofloxacin, metronidazole and minocycline in situ". International Endodontic Journal 29 (1996): 118-124.

5. Hauman $\mathrm{CHJ}$ and Love RM. "Biocompatibility of dental materials used in contemporary endodontic therapy: a review. Part 1. Intracanal drugs and substances". International Endodontic Journal 36 (2003): 75-85.

Volume 2 Issue 8 August 2019

(C) All rights are reserved by Michel Goldberg. 